\title{
Velocity-Density Systematics of Fe-5wt\%Si: Constraints on Si Content in the Earth's Inner Core
}

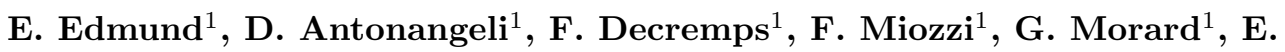 \\ Boulard $^{1}$, A. N. Clark ${ }^{1}$, S. Ayrinhac ${ }^{1}$, M. Gauthier ${ }^{1}$, M. Morand ${ }^{1}$, M. \\ Mezouar $^{2}$ \\ ${ }^{1}$ Institut de Minéralogie, de Physique des Matériaux et de Cosmochimie (IMPMC), Sorbonne Université, \\ UMR CNRS 7590, IRD, Muséum National d'Histoire Naturelle, Paris 75005 France \\ ${ }^{2}$ ESRF, F-38043 Grenoble, France
}

\section{Key Points:}

- Isothermal compression of Fe-5wt.\%Si at high temperatures up to $1.1 \mathrm{Mbar}$ and $2100 \mathrm{~K}$

- Measurement of Vp of Fe-5wt.\%Si under quasihydrostatic conditions to 1.1 Mbar

- Si cannot be the sole light element in the Earth's inner core 


\begin{abstract}
The elasticity of hcp-Fe-5wt\%Si has been investigated by synchrotron X-ray diffraction up to $110 \mathrm{GPa}$ and $2100 \mathrm{~K}$, and by picosecond acoustics measurements at ambient temperature up to $115 \mathrm{GPa}$. The established Pressure-VolumeTemperature (PVT) equation of state shows that the density of the Earths inner core can be matched by an Fe-Si alloy with 5 wt\% Si for all reasonable core temperatures, but that its compressional and shear velocities remain too high with respect to seismological observations. On the other hand, Fe-Si alloys whose velocities are expected to get close to seismological observations are too dense at relevant temperatures. Thus, based on these combined velocity-density measurements, silicon is not likely to be the sole light element in the inner core.
\end{abstract}

\title{
1 Introduction
}

Iron and iron alloys at extreme conditions have garnered significant interest due to their relevance to Earths deep interior. While a first 1D reference seismological model providing density $(\rho)$, compressional $(\mathrm{Vp})$ and shear $(\mathrm{Vs})$ sound velocities as a function of depth into the Earth (PREM Preliminary Reference Earth Model) was established in the 1980s (Dziewonski and Anderson, 1981), there is still considerable debate over the chemical composition of the Earths core. On cosmochemical grounds, iron is considered to be the most abundant element in the Earth's core (e.g., Allégre et al., 1995; McDonough and Sun, 1995). However, early on in the study of Fe at extreme conditions, it was observed that Fe is too dense at the pressure $(\mathrm{P})$ and temperature $(\mathrm{T})$ conditions of the core to be the sole element present (Birch, 1952). Earths solid inner core exists at pressures of 330-360 GPa, and at temperatures of 5000-7000K based on the melting curve of Fe (Anzellini et al., 2013; Boehler, 1993; Nguyen and Holmes, 2004). As a consequence of the density difference between Fe and PREM, there needs to be some quantity of elements lighter than Fe alloyed to it in order to compensate for this density deficit. Among the potential light element candidates, $\mathrm{Si}$ has been favored by many recent studies but without reaching a firm consensus. Regardless of the nature of accretionary materials and redox path, all recent core differentiation models based on metal-silicate partitioning support the presence of Si in the core (Fischer et al., 2015; Siebert et al., 2013). The presence of $\mathrm{Si}$ is also advocated on the basis of isotopic arguments (Fitoussi et al., 2009). The possible presence and the quantity of Si in the Earths core has important implications for geodynamic processes and the bulk redox state of the Earths interior (Hirose et al., 2017; Wade and Wood, 2005; Wood et al., 1990). One possible way to constrain the Si content of the inner core is the comparison between seismological data and experimental measurements, or calculations, of $\rho, \mathrm{Vp}$ and $\mathrm{Vs}$ of candidate materials at pertinent PT conditions (Sakairi et al., 2018; Sakamaki et al., 2016). Based off the measurement of Vp vs. $\rho$ at extreme conditions, estimates have varied from 1-2 wt\% Si (Antonangeli et al., 2010; Badro et al., 2007) to 8 wt\% Si (Fischer et al., 2014; Mao et al., 2012 ), with the most recent measurements putting an upper limit of $5 \mathrm{wt} \% \mathrm{Si}$ (Antonangeli et al., 2018; Sakairi et al., 2018).

Thanks to the adaptation of Picosecond Acoustics (PA) to the Diamond Anvil Cell (DAC), it is possible to make direct measurements of the acoustic travel time of Fe-alloys, and metals in general, at very high pressures (Decremps et al., 2014,0). Additionally, PA has fewer limitations on sample dimensions than conventional synchrotron-based techniques, allowing the measurement of $\mathrm{Vp}$ under quasihydrostatic conditions to Mbar pressures. We thus used PA to probe acoustic echoes and the compressional sound velocity of an Fe-Si alloy with 5wt. \% Si up to $115 \mathrm{GPa}$. We complemented these measurements with synchrotron x-ray diffraction measurements in laser-heated diamond anvil cells up to $110 \mathrm{GPa}$ and $2100 \mathrm{~K}$, deriving a P-V-T equation of state. Our results provide tight constraints on the Si abundance in the Earths inner core. 


\section{Methods}

\subsection{Sample Preparation}

All experiments were performed on an Fe-Si alloy synthesized by melt spinning at ICMPE laboratory in Thiais, France (melt temperature $\mathrm{T}=1823 \mathrm{~K}$, wheel velocity v $=20 \mathrm{~m} / \mathrm{s}$, (Morard et al., 2011)). Scanning electron microscopy measurements have shown the alloy to be chemically homogeneous and contain $5.2(2) \mathrm{wt} \% \mathrm{Si}$, and so the alloy will be referred to hereafter as Fe-5Si for brevity. Fe-5Si was also measured by grazing-incidence $\mathrm{x}$-ray diffraction (XRD) at ambient conditions to check phase purity and density, showing minimal texture and excellent polycrystallinity. Experiments were performed using Le Toullec-type membrane-driven DACs with diamonds of culet size ranging from 250 $\mu \mathrm{m}$ down to $100 \mu \mathrm{m}$ and equipped with Re gaskets. All experiments at ambient temperature were performed with Ne as the pressure-transmitting medium (PTM) to ensure quasihydrostatic conditions up to Mbar conditions. Samples were scraped from a large, thin ribbon, and sample dimensions were chosen such that there was no bridging between sample and diamonds, nor contact between gasket and sample. For PA experiments, the thickness of the sample was determined by the measured initial travel time and derived Vp from literature elastic constants (see 2.3). In PA Run 1 the sample was determined to be 3.73(6) $\mu \mathrm{m}$ thick, and for Run 2 the sample was determined to be 1.84(3) $\mu \mathrm{m}$ thick. For high-T XRD the sample was 1-3 $\mu \mathrm{m}$ thick, and for ambient-T XRD the sample was 5-7 $\mu \mathrm{m}$ thick, estimated by optical microscopy (during sample loading) and observations of diffraction intensity relative to PTM.

\subsection{X-ray Diffraction}

For room temperature measurements, Fe-5Si was loaded alongside Mo (Pressure calibration: $\mathrm{K}_{0}=260(1) \mathrm{GPa}, \mathrm{K}^{\prime}=4.19(5)$, using the reference volume of $\mathrm{Mo}-\mathrm{V}_{0}=$ $31.17 \AA^{3}$, Litasov et al., 2013; Ross and Hume-Rothery, 1963) or Pt $\left(\mathrm{K}_{0}=277.3 \mathrm{GPa}\right.$, $\mathrm{K}^{\prime}=5.12, \mathrm{~V}_{0}=60.38 \AA^{3}$ Dorogokupets and Oganov, 2007) as a pressure calibrant, with $\mathrm{Ne}$ as the PTM. For experiments at high temperatures, the samples were sandwiched between $\mathrm{KCl}$ disks which served as the PTM, and to thermally insulate the sample from the diamonds. $\mathrm{KCl}$ also served as the pressure calibrant. The samples pressure at high temperatures was determined by diffraction of $\mathrm{KCl}$, with the method for estimating temperature following Campbell et al. (2009) and the $\mathrm{KCl}$ pressure calibration of Dewaele et al. (2012). By virtue of its very low thermal pressure, uncertainties on pressure due to thermal gradients across $\mathrm{KCl}$ are below reported error bars. After loading the DAC, the assembly was left open to dry in a vacuum oven at $130^{\circ} \mathrm{C}$ for at least $1 \mathrm{hr}$ after which the DAC was closed. This practice ensures that moisture content is minimal within the sample chamber. Angle-dispersive XRD measurements were performed at beamline ID27 at the ESRF (Mezouar et al., 2005). The radiation was monochromatic $(\lambda=0.3738 \mathrm{~nm})$ focused to approximately $3 \times 3 \mu \mathrm{m}^{2}$ (horizontal x vertical FWHM). Diffraction patterns were collected on a MarCCD camera, with collection times of 30-60 seconds. Samples were heated on both sides by two continuous Nd:YAG fibre lasers (TEM00), each one delivering up to $200 \mathrm{~W}$. Hot spots were approximately $20 \mu \mathrm{m}$ in diameter, much larger than the FWHM of the focused X-ray beam. All temperatures were measured by the spectroradiometric method, using a Planck fit of the observed blackbody radiation from the center of the heating spot, as described by Mezouar et al. (2017). While absolute errors in temperature are on the order of $150 \mathrm{~K}$, the measured temperature was seen to vary by less than $30 \mathrm{~K}$ during pattern integration (averaged over 3-5 measurements per diffraction pattern). Diffraction images were calibrated against a $\mathrm{CeO}_{2}$ standard, and then radially integrated using Dioptas image processing software (Prescher and Prakapenka, 2015). The integrated diffraction pattern was then analysed by use of Le Bail fits in the software Jana2006 (Václav et al., 2014). 


\subsection{Picosecond Acoustics}

These experiments are performed in pump-probe configuration, where laser pulses generated by a Maitai Ti:Sapphire laser $(\lambda=800 \mathrm{~nm}$, pulse duration $=100 \mathrm{fs})$ are separated into two beams which are focused at the two opposing faces of the metallic sample. The majority of the intensity $(\sim 80 \%)$ of the laser is directed towards the pump side (30-100 $\mathrm{mW}$ depending on experimental conditions), where the beam generates a small thermal stress at the surface of the sample. The relaxation of this thermal stress generates an elastic wave which propagates through the sample. The probe beam, comprising the remainder of the lasing intensity, is analysed by means of an interferometer, in order to detect the change of phase of reflectivity at the sample surface. This quantity changes abruptly upon arrival of the acoustic wave, and therefore provides an accurate determination of the acoustic travel time across the material. Further details of the setup are provided elsewhere (Decremps et al., 2015). At ambient conditions, the value of Vp was derived from single crystal elastic constants (Machová and Kadečková, 1977) using the Hashin-Strikman average (Hashin and Shtrikman, 1962) and combined with the measured acoustic travel time to determine initial thickness. The acoustic travel time used to determine the thickness of the sample was an average of several measurements across the sample surface, with the travel time of each location being derived from the time difference between the first and second acoustic echo. This procedure was performed before and after each experiment for each sample. The thickness at pressure was assumed to scale as $\left(V / V_{0}\right)^{1 / 3}$ where $\mathrm{V}_{0}$ is the $\mathrm{V}_{0}$ of bcc Fe-5Si, measured to be $23.34(4)^{3}$ and $\mathrm{V}$ is determined from the fitted EoS for hcp-Fe-5Si. Thickness and travel times as a function of pressure were then combined to determine Vp up to Mbar pressures. Ruby fluorescence (Mao et al., 1986; Sokolova et al., 2013) and the Raman shift of the center of the diamond culet (Akahama and Kawamura, 2006) were used to assess pressure. Determination by ruby spectra and diamond edge Raman were within 2 GPa of each other at all pressures where both were measured. Ruby fluorescence was measured before and after every travel time measurement, and the reported pressure is an average of the two values. For Run 1, the difference in pressures determined from ruby fluorescence before and after measurement was less than $0.5 \mathrm{GPa}$, while for Run 2 it was less than $2 \mathrm{GPa}$. All reported pressures are derived from ruby fluorescence measurements with the calibration of Sokolova et al. (2013).

\section{Results}

\subsection{X-ray Diffraction}

Two runs were performed in Ne at $300 \mathrm{~K}$, one of which used Pt as pressure calibrant to $41 \mathrm{GPa}$, and another run measured to $1.1 \mathrm{Mbar}$ with Mo as the pressure calibrant. Diffraction of the pressure calibrant was collected independently from that of the sample by translating the cell a few microns from the sample position. As sample reflections were also observable at the calibrant position, the presented volumes are unweighted averages of all measured volumes at a given pressure step, and the reported pressure are averages of Mo/Pt measurements taken at the same pressure step, drift between pressure measurements was typically less than $0.2 \mathrm{GPa}$. While measurement error of Mo volume and the statistical errors in the calibrant EoS are small $(<0.5 \%)$, the absolute error at $300 \mathrm{~K}$ due to the intrinsic uncertainty in pressure calibration and pressure gradients is $\sim 2-3 \%$. The bcc-hcp transition started at about $14 \mathrm{GPa}$ and all bcc reflections were absent by $21 \mathrm{GPa}$.

In another set of experiments, Fe-5Si was compressed in a laser-heated membrane DAC along two high-temperature isotherms, at about $1450 \mathrm{~K}$ and at about $2100 \mathrm{~K}$. Temperatures varied by less than $100 \mathrm{~K}$ along each isotherm $(1 \sigma=30 \mathrm{~K}$ at $1450 \mathrm{~K}$ and $1 \sigma$ $=50 \mathrm{~K}$ at $2100 \mathrm{~K}$ ). Temperatures were corrected downwards by around $3 \%$ following standard methods (Campbell et al., 2009) to account for axial T gradients (however this 
had a negligible effect on the fitted equation of state parameters). No phase other than hcp-Fe-5Si was observed at the HP-HT conditions of the present study, consistent with Tateno et al. (2015). Integrated diffraction patterns in Ne and at high temperatures are discussed in Supplementary Text S1 and shown in Supplementary Figures S1 and S2.

The ambient temperature component of the P-V-T thermal model employed in the present study consisted of either a 3rd Order Birch-Murnaghan (Equation 1, Birch, 1947) or Vinet (Equation 2, Vinet et al., 1989) EoS:

$$
\begin{aligned}
P_{300 K}(V)= & \frac{3}{2} K_{0}\left[\left(\frac{V_{0}}{V}\right)^{7 / 3}-\left(\frac{V_{0}}{V}\right)^{5 / 3}\right]\left\{1+\frac{3}{4}\left(K^{\prime}-4\right)\left[\left(\frac{V_{0}}{V}\right)^{2 / 3}-1\right]\right\} \\
& P_{300 K}(V)=3 K_{0}\left(\frac{1-\eta}{\eta^{2}}\right) \exp \left[\frac{3}{2}\left(K^{\prime}-1\right)(1-\eta)\right]
\end{aligned}
$$

Where $\mathrm{V}_{0}, \mathrm{~K}_{0}$ and $\mathrm{K}^{\prime}$ are the unit cell volume $\left(\AA^{3}\right)$, bulk modulus (GPa) and $\frac{d K}{d P}$ at ambient conditions. In Equation 2 specifically, $\eta=\left(\frac{V}{V_{0}}\right)^{(1 / 3)}$

The thermal parametrization is shown in Equation 3.

$$
P(V, T)=P_{300 K}(V)+\left.P_{v i b}(V, T)\right|_{300} ^{T}+\left.P_{e l+a n h}(V, T)\right|_{300} ^{T}
$$

In Equation 3, $P_{v i b}(V, T)$ is given by:

$$
P_{v i b}(V, T)=\frac{9 N R \gamma_{v i b}}{V}\left[\frac{\theta_{D}}{8}+T\left(\frac{T}{\theta_{D}}\right)^{3} \int_{300}^{\theta_{D} / T} \frac{x^{3}}{\exp (x)-1} d x\right]
$$

Where $\gamma_{v i b}$ is the vibrational Grüneisen parameter, $\theta_{D}$ is the Debye temperature, $\mathrm{N}$ is the number of atoms per formula unit ( $\mathrm{N}=2$ for hcp-structured Fe-alloys). $\mathrm{R}$ is the ideal gas constant, $\mathrm{V}$ is unit cell volume (in units of $\mathrm{cm}^{3} / \mathrm{mol}$ ) and $\mathrm{T}$ is the temperature (in K). The volume dependence of the vibrational Grüneisen parameter and Debye temperature are given by Equations 5 and 6 respectively.

$$
\begin{gathered}
\left(\frac{\gamma_{v i b}}{\gamma_{v i b, 0}}\right)=\left(\frac{V}{V_{0}}\right)^{q} \\
\theta_{D}=\theta_{D, 0} \exp \left[\left(\gamma_{v i b, 0}-\gamma_{v i b}\right) / q\right]
\end{gathered}
$$

In Equations 5 and 6 q characterizes the volume dependence of the vibrational contributions to thermal pressure. In the fitting process, $\theta_{D, 0}$ was fixed to $422 \mathrm{~K}$.

Due to the similar $\mathrm{T}^{2}$ dependence of $\mathrm{P}_{e l}$ and $\mathrm{P}_{a n h}$, a single electronic pressure term was used for fitting in the present thermal model:

$$
P_{e l}(V, T)=\frac{\gamma_{e}}{V} \beta_{0}\left(\frac{V}{V_{0}}\right)^{k} T^{2}
$$

Where $\gamma_{e}$ is the electronic Grüneisen parameter (here fixed to 2 after (Fei et al., 2016)), $\beta_{0}$ is the electronic heat capacity and $\mathbf{k}$ (fixed to 1.34) is an exponent which characterizes the volume dependence of the electronic contribution to thermal pressure. 
All parameters of the presented P-V-T EoS except $\mathrm{k}, \gamma_{e}$ and $\theta_{D}$ were refined simultaneously by an unweighted least-squares fit to the entire dataset. When the Debye temperature is fixed in literature, it is generally fixed to anywhere between 417-422 K for Fe and Fe-Si alloys (e.g. Dewaele et al., 2006; Fischer et al., 2012,1), however such differences are negligible with regards to the resultant EoS. Additionally, most of the studies which have constrained $\mathrm{k}$ either experimentally or by means of ab initio calculations (Boness et al., 1986; Dewaele et al., 2006; Fei et al., 2016) generally report a k value of $\sim 1.34$. Varying $\mathrm{k}$ on the order of $\sim 0.1$ in the fitting process produces deviations in extrapolated densities at inner core conditions of less than $0.2 \%$, far below the error bar of the extrapolation $(\sim 1.5 \%)$. It is stressed that while electronic contributions to thermal pressure are relatively small (up to $\sim 5 \mathrm{GPa}$ at $2100 \mathrm{~K}$ ), it was not possible to fit the present dataset to a purely vibrational model of thermal pressure, as such a model could not simultaneously reproduce the compressional behaviour of both the $1450 \mathrm{~K}$ and $2100 \mathrm{~K}$ isotherms. The fitted EoS parameters of this dataset are shown in Table 1. All datapoints were within $2 \mathrm{GPa}$ of the fitted EoS, irrespective of whether a 3BM or Vinet EoS was used for the ambient temperature compression curve.

At ambient temperature, the present dataset is similar to recent measurements of Fe10Ni5Si (Morrison et al., 2018) and those of hcp-Fe in He (Dewaele et al., 2006). Discrepancies between the presented ambient temperature EoS parameters are primarily due to small systematic differences observed at low pressures (below $~ 60 \mathrm{GPa}$ ), as Fe, Fe10Ni5Si and Fe5Si all have nearly identical volumes by 1 Mbar. Shown in Figure 1 as raw datapoints, the presented alloy exhibits a slightly higher volume than hcp-Fe, consistent with literature on Fe-Si alloys (Fischer et al., 2014; Lin et al., 2003a; Tateno et al., 2015). While the Earth's core is likely to be composed of an Fe-Ni alloy, the addition of Ni likely does not significantly change the presented results and conclusions, due to the weak effect that $\mathrm{Ni}$ has on Vp relative to Fe and Si (Antonangeli et al., 2010; Liu et al., 2016; Martorell et al., 2013; Wakamatsu et al., 2018) and its weak effect on density (Morrison et al., 2018). However it is stressed that at present there are few constraints on the thermoelastic behaviour of Fe-Ni or Fe-Ni-Si alloys at simultaneous high P-T conditions.

It is immediately noticeable in Fig. 2 that the P-V-T EoS of Fe-5Si measured here is similar to that of reported thermal parametrizations of hcp-Fe (Dewaele et al., 2006; Fei et al., 2016), considering the small differences in $300 \mathrm{~K} \mathrm{EoS} \mathrm{in} \mathrm{each} \mathrm{study.} \mathrm{Unsur-}$ prisingly, the fitted P-V-T EoS parameters of Fe-5Si are in good agreement with those of Fei et al. (2016), which employs the same type of thermal model. It is remarkable that the present XRD dataset composed purely of static compression data is capable of producing a $\mathrm{P}-\mathrm{V}-\mathrm{T}$ EoS which is directly comparable to those incorporating extensive parametrization using shock compression data, ab initio calculations and/or NRIXS (e.g. Dewaele et al., 2006; Fei et al., 2016).

As the direct measurement of thermal EoS are at the cutting edge of experimental capabilities, it has been common in recent past to use $a b$ initio parametrizations to account for $\mathrm{P}_{e l}$ (e.g. Dewaele et al., 2006). Inputs from calculations have been used to constrain $\mathrm{P}_{e l}$ and fit a purely vibrational model (Yamazaki et al., 2012), or to construct thermal models using purely ambient temperature experimental data (e.g. Lin et al., 2003a; Morrison et al., 2018). Choice of parametrization can change electronic thermal pressures at core conditions by nearly $\mathbf{5 0 \%}$, and $\mathrm{P}_{e l}$ itself is comparable in magnitude to $\mathrm{P}_{v i b}$ at such conditions. Rescaling $\mathrm{P}_{e l}+\mathrm{P}_{a n h}$ of Dewaele et al. (2006) to the formalism used in the present study, $\beta_{0} \approx 1.7$. As the fitted $\beta_{0}$ of the present work is $\sim 3.2(9)$ and that of Fei et al. (2016) is 3.91, it is likely for a dilute Fe-alloy (of realistic composition for the Earth's inner core) that $\mathrm{P}_{e l}$ is larger than that reported by Dewaele et al. (2006). 


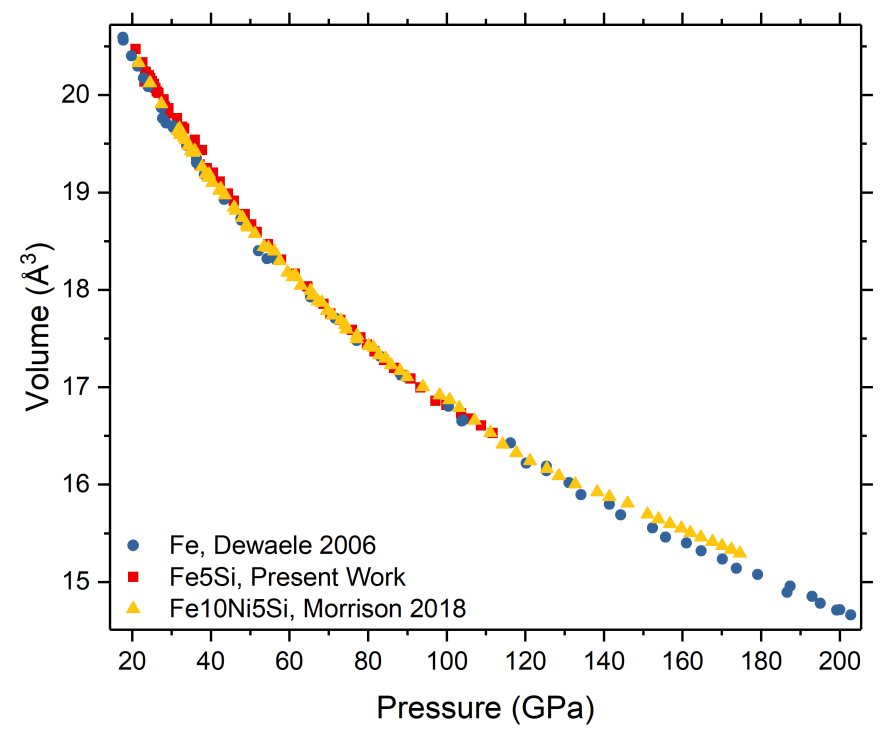

Figure 1. Ambient temperature P-V relations of Fe-5Si (present work), pure Fe (Dewaele et al., 2006) and Fe10Ni5Si (Morrison et al., 2018). It is observed that Fe5Si and Fe10Ni5Si exhibit a small systematic difference up to $60 \mathrm{GPa}$, with their volumes becoming indistinguishable at higher pressures.

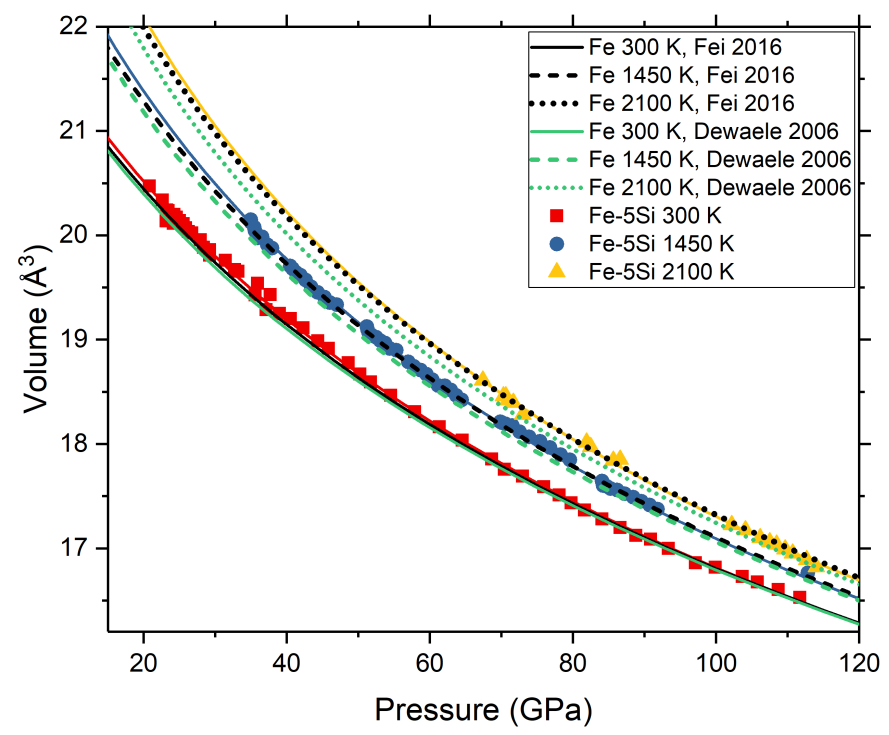

Figure 2. P-V-T dataset measured in the present study. Shown as lines are the results of the 3BM EoS fit described here, and the P-V-T relations reported by literature on hcp-Fe (Dewaele et al., 2006; Fei et al., 2016). 


\begin{tabular}{|c|c|c|c|c|c|}
\hline & Fe5Si & Fe5Si & Fe F16 & Fe Y12 & Fe9Si F14 \\
\hline Formalism & $3 \mathrm{BM}$ & Vinet & $3 \mathrm{BM}$ & $3 \mathrm{BM}$ & $3 \mathrm{BM}$ \\
\hline$V_{0}$ & $22.524(62)$ & $22.587(67)$ & $\mathbf{2 2 . 4 2 8}$ & $22.15(5)$ & $23.92(18)$ \\
\hline$K_{0}$ & $172.4(6.0)$ & $163.3(6.9)$ & $172.7(1.4)$ & $202(7)$ & $129.1(1.4)$ \\
\hline$K^{\prime}$ & $4.64(14)$ & $5.13(16)$ & $4.79(0.05)$ & $4.5(2)$ & $5.29(8)$ \\
\hline$\theta_{D}$ & $\mathbf{4 2 2}$ & $\mathbf{4 2 2}$ & $\mathbf{4 2 2}$ & $1173(62)$ & $\mathbf{4 2 0}$ \\
\hline$\gamma_{0}$ & $1.72(13)$ & $1.73(13)$ & $\mathbf{1 . 7 4}$ & $3.2(2)$ & $1.14(14)$ \\
\hline$q$ & $0.65(23)$ & $0.67(23)$ & $\mathbf{0 . 7 8}$ & $0.8(3)$ & $\mathbf{1}$ \\
\hline$\beta_{0}$ & $3.20(85)$ & $3.22(85)$ & 3.91 & ab initio & - \\
\hline$k$ & $\mathbf{1 . 3 4}$ & $\mathbf{1 . 3 4}$ & 1.34 & ab initio & - \\
\hline
\end{tabular}

Table 1. $V_{0}$ has units of $\AA^{3}, \mathrm{~K}_{0}$ has units of $\mathrm{GPa}, \theta_{D}$ has units of $\mathrm{K}, \beta_{0}$ has units of $\mathrm{cm}^{3}$ $\mathrm{mol}^{-1} \mathrm{JK}^{-2} 10^{-6}$, the other parameters are dimensionless. F16 denotes (Fei et al., 2016), Y12 denotes (Yamazaki et al., 2012), and F14 denotes (Fischer et al., 2014). Parameters in bold font are those which have been fixed during the fitting process. The two thermal models of Fe5Si are based on different choices of ambient temperature EoS formalism.

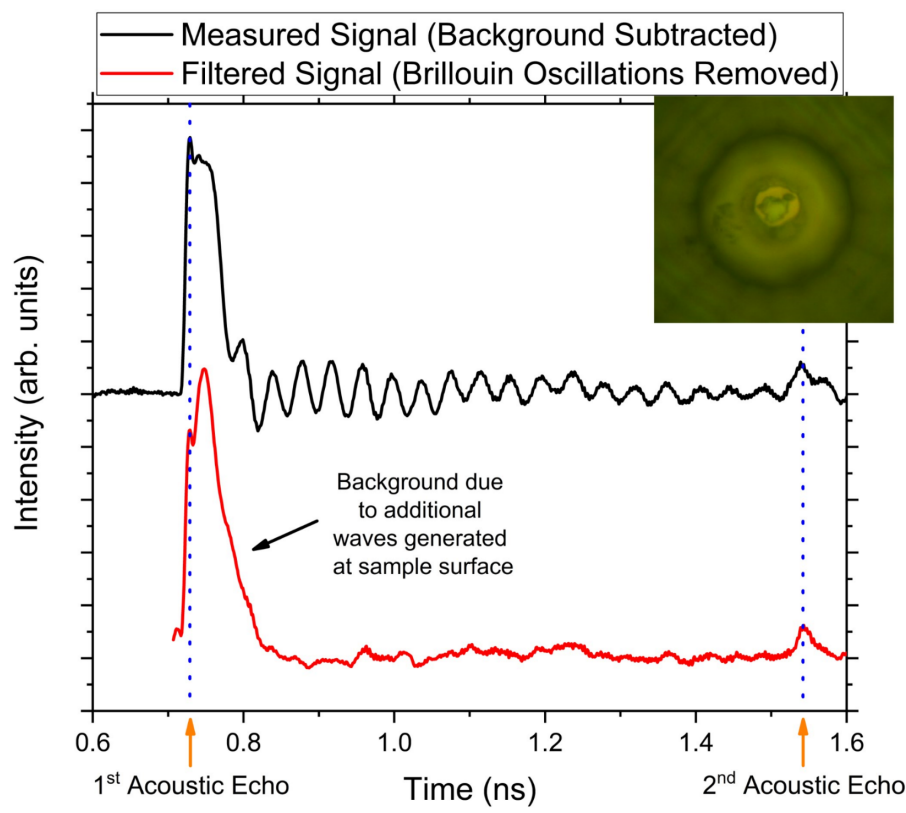

Figure 3. Travel Time measurement by Picosecond Acoustics at $47 \mathrm{GPa}$. The sharp peak associated with the 1st acoustic echo is clearly visible, followed by a background signal related to the generation of surface waves and Brillouin scattering in the Ne PTM (filtered in the red curve). The difference between the travel time of filtered and unfiltered data changes by a maximum of $2 \mathrm{ps}$, or an error of $0.1-0.5 \%$ of $\mathrm{Vp}$ depending on sample thickness. Inset is the sample chamber of Run 2 at $\sim 100 \mathrm{GPa}$. 


\subsection{Picosecond Acoustics}

Travel times were measured as a function of pressure (Figure 3 and Figure 4 ) in two independent runs performed on samples of Fe-5Si alloy of different initial thickness. Errors in Vp were about $2 \%$ up to $60 \mathrm{GPa}$, and about $3 \%$ by $115 \mathrm{GPa}$. Shown in Figure 3 are background-subtracted time domain signals at $47 \mathrm{GPa}$ before and after data treatment to extract Brillouin oscillations. Larger errors at high pressures are due to an increase in diffuse background caused by the progressive depolarization of the pump and probe beams resulting from increased defect scattering, stress gradients across the diamond anvil, and the cupping of the diamond culet- classical issues encountered by optical measurements at Mbar conditions (Merkel et al., 1999). Uncertainties of sample thickness, especially at high pressure, has only a small effect on the travel time compared to the change in velocity. As a matter of fact, by 1 Mbar, the thickness has changed by $\sim 10 \%$ relative to ambient pressure, while the acoustic travel time is typically $50 \%$ its original value. Sample tilt within the sample chamber has a negligible effect on measured travel times due to the instrumental configuration and focusing strategy employed. In Run 1, it was seen that there were some residual stresses in the sample which induced local variation in measured travel times of about 2-3\% in the bcc phase, and so for Run 2, the sample was additionally annealed under vacuum at $\sim 400 \mathrm{~K}$ for $12 \mathrm{~h}$. This procedure effectively reduced scatter in measured travel times to less than $1 \%$ in the bcc phase. Normalized travel times for bcc and hcp Fe5Si are shown in Figure 4.

In Run 2, the initial travel time and travel time of the recovered samples are within error bar of each other, indicating negligible plastic deformation of the sample when compressed in Ne PTM up to 1.1 Mbar. Provided the volume decrease at the bcc-hcp transition is accounted for, the measured Vp varies by less than $0.5 \%$ depending on the choice of Fe or Fe-Si EoS, well within error at all pressures. The bcc-hcp transition is observed by PA to occur over a pressure range in close agreement with XRD measurements.

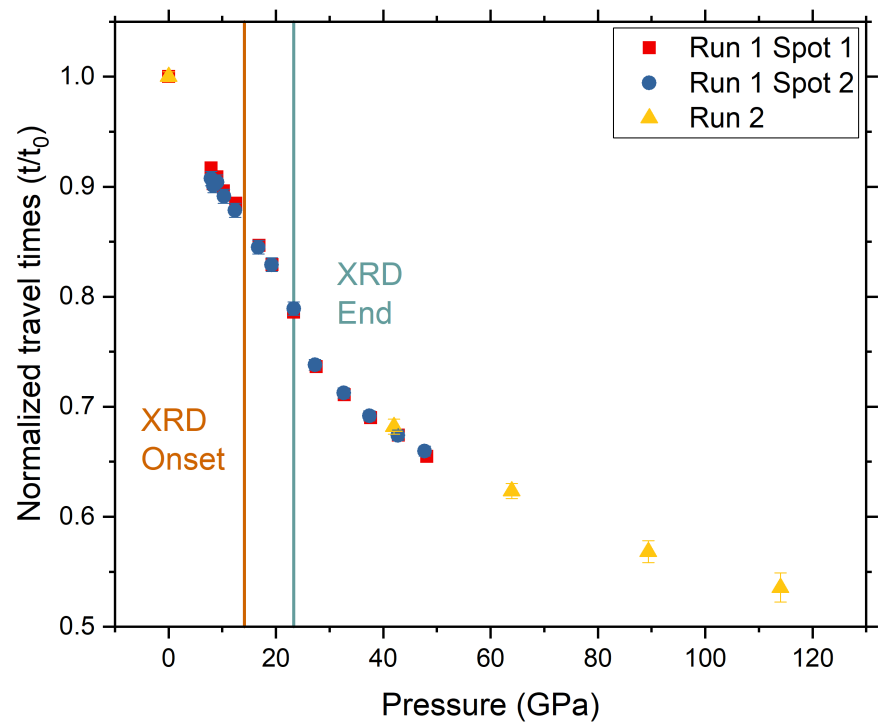

Figure 4. Normalized travel times of Fe-5Si from $~ 5-115$ GPa. Observed scatter in the bccphase disappears above the bcc-hcp transition. The onset of the bcc-hcp transition measured by $\mathrm{PA}$ is in good agreement with that observed by XRD. 
Following the transition region determined by both XRD and PA, a sharp rise in $\mathrm{Vp}$ is observed. While bcc peaks are absent from XRD by $\sim \mathbf{2 3} \mathbf{~ G P a}$, nonlinear variations in acoustic velocity have been observed up to $\sim 30 \mathrm{GPa}$ where the variation becomes regular. This is attributed to the slight development of preferred orientations in hcp-Fe$5 \mathrm{Si}$ at the end of the phase transition - XRD highlights a moderate change in the intensity ratio of the $(002) /(100)$ peaks between 20 to $30 \mathrm{GPa}$, while at higher pressures this ratio does not vary any more. Due to the difference in measurement geometry between PA and XRD, the intensity reduction of the (002) peak observed by XRD reflects a larger contribution of the c-axis to the measured travel time and hence a small increase in $\mathrm{Vp}$ (Antonangeli et al., 2006). As linearity in the $\mathrm{Vp}-\rho$ relation for hcp-Fe-5Si was observed by $\sim 30 \mathrm{GPa}$, the following discussion will be limited to data measured at and above this pressure. 


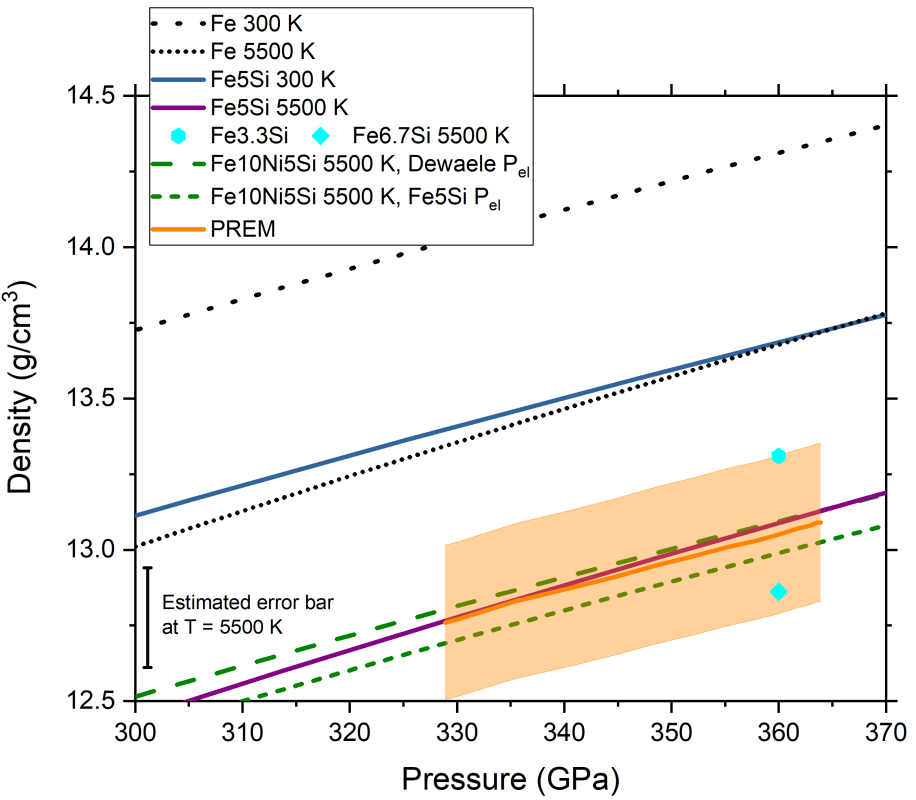

Figure 5. Density of Fe (Fei et al., 2016), Fe-5Si, Fe-10Ni-5Si (Morrison et al., 2018), Fe3.3Si and Fe-6.7Si (Martorell et al., 2016) at the pressures of the IC. Fe-5Si is within error bar of PREM for all reasonable core temperatures (5000-7000 K). Shaded region denotes expected error bar of PREM ( 2\%, Masters and Gubbins, 2003). Extrapolations of Fe-5Si to core temperatures and pressures have an error bar of $\sim 1.5 \%$.

\section{Discussion}

4.1 Density of Fe-Si Alloys at the P-T Conditions of Earth's Inner Core

Extrapolated to IC pressures and temperatures, the density of Fe-5Si is within error of PREM ( $\sim 2 \%$, Masters and Gubbins, 2003) for all reasonable core temperatures, with the best match for $\mathrm{T}=5500 \mathrm{~K}$ (Fig. 5). As the compressional behaviour of Fe-5Si and $\mathrm{Fe}$ are similar over the wide range of $\mathrm{P}-\mathrm{T}$ conditions measured in this study, the dominant mechanism for density reduction even at core conditions is simply the difference in atomic mass between Fe and Si. Indeed, the density reduction between hcp-Fe and hcp-Fe-5Si is similar both at $300 \mathrm{~K}$ and at temperatures exceeding $5000 \mathrm{~K}$. While the recent measurements of Fe-10Ni-5Si exhibit less compressible behaviour at ambient temperature, extrapolations of that EoS to core conditions results in densities very similar to those presented here. It is noted however that updating the Fe-10Ni-5Si thermal model with $\mathrm{P}_{e l}$ determined here, there is a $\sim 0.1 \mathrm{~g} \mathrm{~cm}^{-3}(0.8 \%)$ decrease in density of the alloy at $5500 \mathrm{~K}$. This highlights the importance of accurate, high quality volume measurements at simultaneous high temperatures and high pressures, in order to constrain such effects at the conditions of the Earth's inner core.

Our results are well compatible with the most recent ab initio calculations on FeSi alloys (Li et al., 2018; Martorell et al., 2016), the $5500 \mathrm{~K}$ isotherm extrapolates to the midpoint between calculations of Fe-3.3Si and Fe-6.7Si at the same temperatures. The calculated thermodynamic grüneisen parameter of this alloy is between 1.5 and 1.6 at the ICB for temperatures of between $4500 \mathrm{~K}$ and $6500 \mathrm{~K}$, consistent with previous results on hcp-Fe (Dewaele et al., 2006; Fei et al., 2016; Vočadlo et al., 2003). This combined with the observation that Si alloying does not strongly affect the melting curve 
of iron (e.g. Fischer et al., 2013; Morard et al., 2017) indicates that Si-alloying would not likely have a strong effect on the thermal profile of the inner core.

\subsection{Sound Velocities of Fe-Si Alloys at Core Conditions}

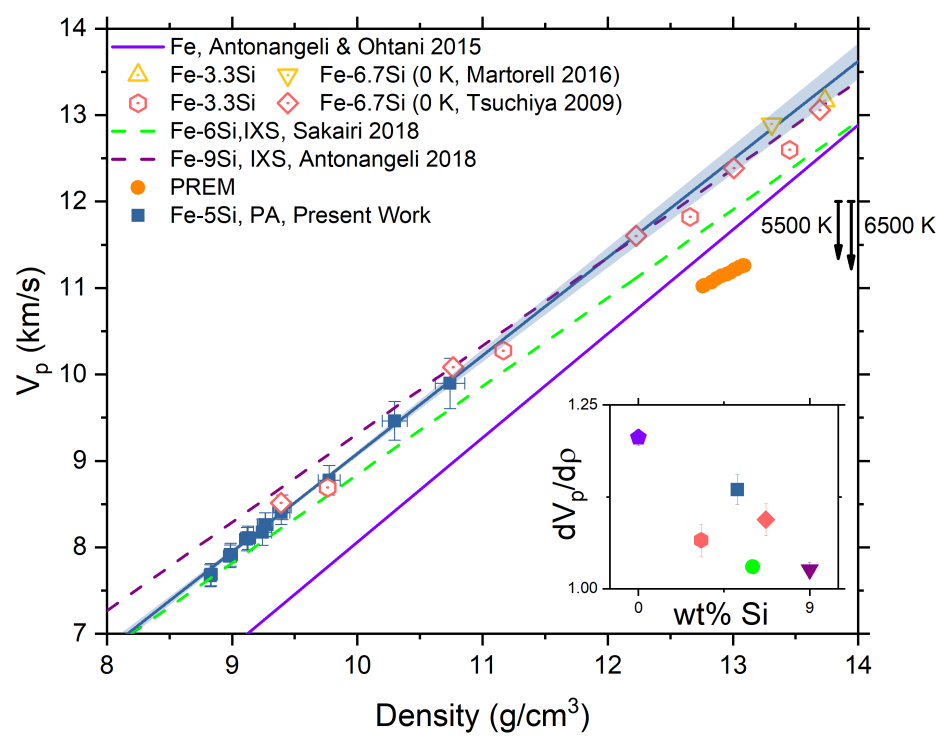

Figure 6. Compressional Sound Velocity vs. Density for Fe and Fe-Si alloys in the hcp structure. Inset: the slope of linear fits to Velocity vs. Density $(\mathbf{d V p} / \mathbf{d} \rho)$ vs. Si content for the here-discussed Fe-xSi datasets (Antonangeli et al., 2018; Mao et al., 2012; Martorell et al., 2016; Sakairi et al., 2018; Tsuchiya and Fujibuchi, 2009). Downward arrows indicate the maximum estimated magnitude of anharmonic effects at $5500 \mathrm{~K}$ and $6500 \mathrm{~K}$ (after Martorell et al., 2016).

Vp measurements show a clear linear trend for the entire density range studied, with the fitted parameters $V p(\mathrm{~km} / \mathrm{s})=1.135(20) * \rho\left(\mathrm{g} / \mathrm{cm}^{3}\right)-2.27(19)$ as shown in Figure $6 . \mathrm{dVp} / \mathrm{d} \rho$ of $\mathrm{Fe}-5 \mathrm{Si}$ is reduced with respect to hcp-Fe Antonangeli and Ohtani (2015), although the effect is not as large as that reported by previous IXS measurements on samples with higher Si content (Antonangeli et al., 2018; Mao et al., 2012; Sakairi et al., 2018), as shown in the inset of Figure 6.

Our measurements extrapolate at inner core densities somewhat higher than recent measurements by IXS on more Si-rich samples (Antonangeli et al., 2018; Mao et al., 2012; Sakairi et al., 2018), but are in very good agreement with the result of athermal ab initio calculations on Fe-3.3Si and Fe-6.7Si (Martorell et al., 2016). We also observe agreement between $\mathbf{V p}-\rho$ relations presented here and those reported in Tsuchiya and Fujibuchi (2009). We stress however, that the agreement between our experiments and calculations worsen when looking at velocities versus pressure, in particular with respect to Tsuchiya and Fujibuchi (2009). In that study, at $40 \mathrm{GPa}$ a linear interpolation of Fe3.3Si and Fe6.7Si exhibits a bulk modulus which is $28 \%$ higher than one derived from the present EoS, and the reported density is $4.5 \%$ higher. 
Fig. 6 shows that IXS results are generally parallel to each other (Antonangeli et al., 2018; Mao et al., 2012; Sakairi et al., 2018), but in disagreement with the present work. It is evident based on the combined results of (Antonangeli et al., 2018; Mao et al., 2012; Sakairi et al., 2018; Tsuchiya and Fujibuchi, 2009), and the results of the present study that $\mathrm{dVp} / \mathrm{d} \rho$ decreases with increasing Si content (shown inset in Fig. 6). While a linear decrease in $\mathrm{dVp} / \mathrm{d} \rho$ with $\mathrm{Si}$ content can rationalize a significant amount of the difference between PA and IXS, there are also systematic differences due to the different measurement geometries of the two techniques. PA measures acoustic travel times along the compression axis of the DAC, and as the sample is expected to develop texture, PA will preferentially sample the c-axis of the alloy. By contrast, IXS measures phonon dispersions perpendicular to the DAC compression axis, and as such preferentially samples the basal plane of the Fe-alloy upon development of texture. In this way, textural effects bias PA and IXS measurements in opposite directions. Additionally, IXS measurements require larger sample volumes, and so are often measured in a solid PTM (Sakairi et al., 2018; Sakamaki et al., 2016) or no PTM at all (Antonangeli et al., 2018). We note however, that the difference in extrapolations of PA and IXS measurements here observed for the Fe-Si system is much more significant than for the case of hcp-Fe (Antonangeli and Ohtani, 2015, references therein).

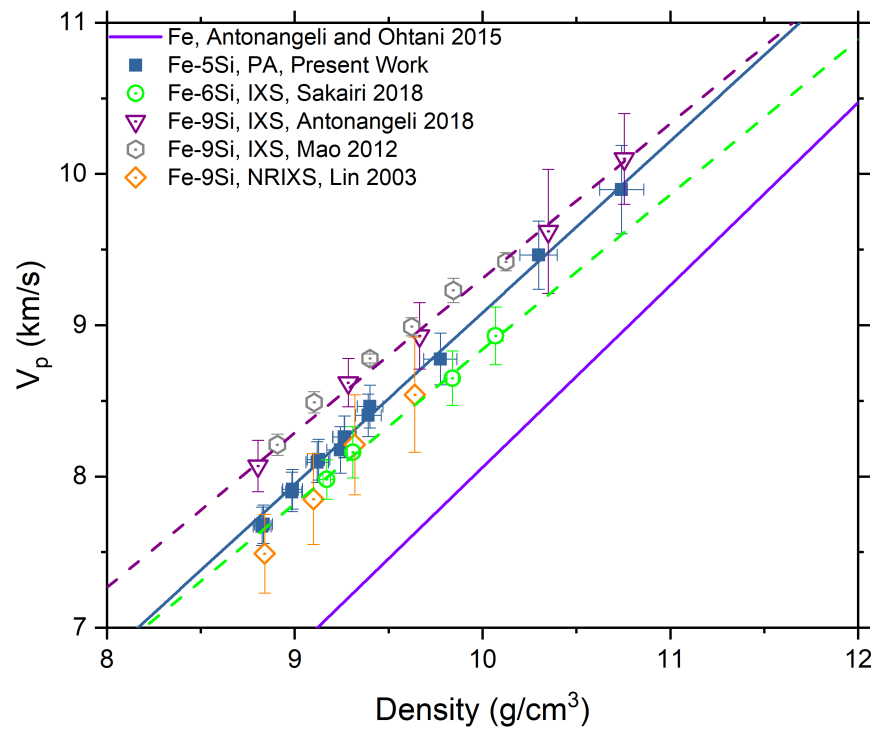

Figure 7. Vp vs Density measured for Fe-Si alloys in the hcp structure by different experimental techniques (Antonangeli et al., 2018; Lin et al., 2003b; Mao et al., 2012; Sakairi et al., 2018).

It is important to minimize texture by performing the experiments under quasihydrostatic conditions with noble gas media. While there is still some development of texture under compression in $\mathrm{Ne}$, it is significantly weaker than the texture observed to occur in IXS experiments at comparable pressure conditions (Supplemental Figure S1,S3, Antonangeli et al., 2018; Sakairi et al., 2018). Figure 7 shows the individual datapoints of various studies in the hcp-Fe-Si system. Ultimately, the effect of preferred orientation is a relatively small effect, but is shown to systematically bias extrapolations (upward in the case of NRIXS) and downwards (in the case of IXS) in Vp at core densities, which strongly hinders compositional modelling at core conditions. It can also be observed in 
Figure 7 that the present work has significantly improved data coverage to typical IXS or NRIXS experiments, and is measured over an extremely wide density range, under quasihydrostatic conditions, allowing for more robust extrapolations to core densities.

Having experimentally determined both the P-V-T EoS and Vp of Fe5Si, it is possible to combine these quantities to derive shear velocities. Figure 8 shows Vs plotted against density. Considering the model-dependence and large error bars of the present shear velocities $(\mathbf{8 - 1 0 \%})$, it is not unambiguously possible to determine whether these vary linearly or sublinearly with density (solid black lines). However, whether the dataset is fit with a linear relation or power-law relation as suggested by (Mao et al., 2012), Vs of Fe5Si is within error of $a b$ initio calculations and seems higher than hcp-Fe (Antonangeli and Ohtani, 2015) at inner core densities. Recent IXS measurements on Fe9Si report a sublinear density dependence of Vs (Antonangeli et al., 2018), indicating that this may be a systematic effect of Si alloying. Shear moduli calculated by Tsuchiya and Fujibuchi (2009) are very similar to the present study at 40 and $120 \mathrm{GPa}$, and the discrepancies observed in Figure 8 arise primarily from the aforementioned differences in densities.

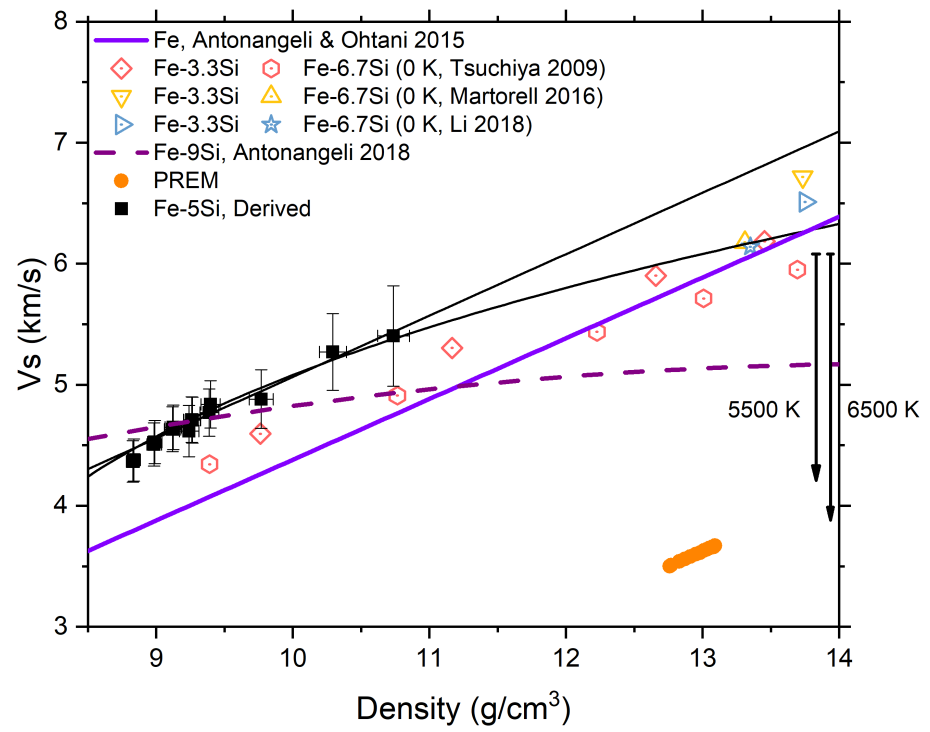

Figure 8. Derived Vs from the present study and selected literature (Antonangeli et al., 2018; Antonangeli and Ohtani, 2015; Li et al., 2018; Martorell et al., 2016; Tsuchiya and Fujibuchi, 2009). It is observed that the derived Vs of Fe5Si are higher than hcp-Fe at core densities irrespectively of the linear or sub-linear extrapolation (solid black lines). Downward arrows indicate the maximum estimated magnitude of anharmonic effects at $5500 \mathrm{~K}$ and $6500 \mathrm{~K}$ (after Martorell et al., 2016).

To meaningfully compare obtained Vp and Vs with PREM, high temperature effects have to be accounted for. At a constant density of $13 \mathrm{~g} / \mathrm{cm}^{3}$, the $\mathrm{T}$ corrections on Vp after experiments by Sakamaki et al. (2016) (on hcp-Fe) and Sakairi et al. (2018) (on Fe-6Si) yield $\mathrm{Vp}$ reductions of $\sim 0.09 \mathrm{~m} \mathrm{~s}^{-1} \mathrm{~K}^{-1}$, with almost no difference between Fe and Fe-6Si. Alternatively, by converting the constant pressure simulations of Martorell et al. (2016) to a constant density of $13 \mathrm{~g} / \mathrm{cm}^{3}$, it is possible to estimate the mag- 
nitude of anharmonic temperature effects to be $\sim 0.11 \mathrm{~m} \mathrm{~s}^{-1} \mathrm{~K}^{-1}$ for Fe and Fe3.3Si, and $\sim 0.05 \mathrm{~m} \mathrm{~s}^{-1} \mathrm{~K}^{-1}$ for Fe-6.7Si.

Regardless of the magnitude of anharmonic corrections for Vp at high temperature (Li et al., 2018; Martorell et al., 2016; Sakairi et al., 2018; Sakamaki et al., 2016), even when assuming the largest proposed effects (Martorell et al., 2016), PREM $\mathrm{Vp}$ is expected to be matched by an Fe- Si alloy only when containing $<2 \mathrm{wt} \% \mathrm{Si}$ for $\mathrm{T}=6500 \mathrm{~K}$ and $<1 \mathrm{wt} \% \mathrm{Si}$ for $\mathrm{T}=5500 \mathrm{~K}$. The largest anharmonic effects reported in recent literature come from ab initio calculations (Martorell et al., 2016), but a more recent ab initio study by the same group using larger simulation cells (Li et al., 2018) supports significantly reduced anharmonic effects compared to Martorell et al. (2016), such that the magnitude of anharmonic effects are more in line with those observed by IXS (Sakairi et al., 2018; Sakamaki et al., 2016). As such, constraints imposed by compressional sound velocity (at maximum $<2 \mathrm{wt} \% \mathrm{Si}$ ) are incompatible with constraints imposed by density (an Si alloy containing $5 \mathrm{wt} \% \mathrm{Si}$ has a density matching PREM at realistic core conditions). As a result, Si likely cannot be the sole light element in the Earths core.

This mismatch between mineral-physics measurements and seismological observables is even worse when considering Vs. Assuming a power-law extrapolation of Vs to core densities (representing a lower bound to the extrapolated Vs) and the largest possible magnitude of anharmonic effects $\left(\sim 0.34 \mathrm{~m} \mathrm{~s}^{-1} \mathrm{~K}^{-1}\right.$ (Martorell et al., 2016)), the Vs of an Fe-Si alloy of any composition cannot match PREM for temperatures below $6500 \mathrm{~K}$. Considering that such high core temperatures are likely to be unrealistic for a variety of geophysical and mineralogical reasons, this emphasizes the unsuitability of Si as the primary light element in the Earth's inner core. This study highlights the important point that density or velocity information alone can only be used to exclude possible core compositions, and must be coupled together in order to develop accurate models of the Earths interior.

\section{Conclusions}

The combination of PVT X-ray diffraction measurements and $300 \mathrm{~K}$ picosecond acoustics Vp measurements under quasi-hydrostatic conditions of Fe-5wt\% Si show that while an alloy with 5 wt\% Si can potentially match the PREM density of the Earths inner core, this quantity of silicon is incompatible with seismological observations of Vp and Vs, even when accounting for anharmonic effects at high temperature. Thus, our paper supports the conclusion suggested by Martorell et al. (2016) that Si cannot be considered as the only light element in the Earth's inner core in the absence of other mechanisms, such as partial melting (Vočadlo, 2007) which significantly reduces density as well as sound velocities of Fe-Si alloys at core conditions.

\section{Acknowledgments}

This work was supported by the Investissements d'Avenir programme (reference ANR11-IDEX-0004-02) and more specifically within the framework of the Cluster of Excellence MATriaux Interfaces Surfaces Environnement (MATISSE) led by Sorbonne Université (grant to DA and FD). Femtosecond laser micro-machining at the Institut de Minéralogie, de Physique des Matériaux et de Cosmochimie (IMPMC), Paris, has been developed and realized by the "Cellule Projet" with the financial support of ANR 2010-JCJC-604-01 (grant to DA). The authors wish to thank Jeroen Jacobs for technical assistance at the ESRF. All data used in the present work can be found in Supplementary Datasets S1S3. 


\section{References}

Akahama, Y. and Kawamura, H.Pressure calibration of diamond anvil Raman gauge to 310GPa, (2006), Journal of Applied Physics, 100(4):043516, $10.1063 / 1.2335683$.

Allégre, C. J., Poirier, J.-P., Humler, E., and Hofmann, A. W.The chemical composition of the Earth, (1995), Earth and Planetary Science Letters, 134(3):515 526, https://doi.org/10.1016/0012-821X(95)00123-T.

Antonangeli, D., Merkel, S., and Farber, D. L.Elastic anisotropy in hep metals at high pressure and the sound wave anisotropy of the Earth's inner core, (2006), Geophysical Research Letters, 33(24):L24303, 10.1029/2006GL028237.

Antonangeli, D., Morard, G., Paolasini, L., Garbarino, G., Murphy, C. A., Edmund, E., Decremps, F., Fiquet, G., Bosak, A., Mezouar, M., and Fei, Y.Sound velocities and density measurements of solid hcp-Fe and hcp-Fe-Si (9 wt.inner core, (2018), Earth and Planetary Science Letters, 482:446 - 453, https://doi.org/10.1016/j.epsl.2017.11.043.

Antonangeli, D. and Ohtani, E.Sound velocity of hcp-Fe at high pressure: experimental constraints, extrapolations and comparison with seismic models, (2015), Progress in Earth and Planetary Science, 2(1):3, 10.1186/ s40645-015-0034-9.

Antonangeli, D., Siebert, J., Badro, J., Farber, D. L., Fiquet, G., Morard, G., and Ryerson, F. J.Composition of the Earth's inner core from high-pressure sound velocity measurements in Fe-Ni-Si alloys, (2010), Earth and Planetary Science Letters, 295(1):292 - 296, https://doi.org/10.1016/j.epsl.2010.04.018.

Anzellini, S., Dewaele, A., Mezouar, M., Loubeyre, P., and Morard, G.Melting of Iron at Earth's Inner Core Boundary Based on Fast X-ray Diffraction, (2013), Science, 340(6131):464-466, 10.1126/science.1233514.

Badro, J., Fiquet, G., Guyot, F., Gregoryanz, E., Occelli, F., Antonangeli, D., and d'Astuto, M.Effect of light elements on the sound velocities in solid iron: Implications for the composition of Earth's core, (2007), Earth and Planetary Science Letters, 254(1):233 - 238, https://doi.org/10.1016/j.epsl.2006.11.025.

Birch, F.Finite Elastic Strain of Cubic Crystals, (1947), Phys. Rev., 71:809-824, 10.1103/PhysRev.71.809.

Birch, F.Elasticity and constitution of the Earth's interior, (1952), Journal of Geophysical Research, 57(2):227-286, 10.1029/JZ057i002p00227.

Boehler, R.Temperatures in the Earth's core from melting-point measurements of iron at high static pressures, (1993), Nature, 363:534-.

Boness, D. A., Brown, J., and McMahan, A.The electronic thermodynamics of iron under Earth core conditions, (1986), Physics of the Earth and Planetary Interiors, 42(4):227 - 240, https://doi.org/10.1016/0031-9201(86)90025-7.

Campbell, A. J., Danielson, L., Righter, K., Seagle, C. T., Wang, Y., and Prakapenka, V. B.High pressure effects on the iron-iron oxide and nickel-nickel oxide oxygen fugacity buffers, (2009), Earth and Planetary Science Letters, 286(3):556 - 564, https://doi.org/10.1016/j.epsl.2009.07.022.

Decremps, F., Antonangeli, D., Gauthier, M., Ayrinhac, S., Morand, M., Marchand, G. L., Bergame, F., and Philippe, J.Sound velocity of iron up to $152 \mathrm{GPa}$ by picosecond acoustics in diamond anvil cell, (2014), Geophysical Research Letters, 41(5):1459-1464, 10.1002/2013GL058859.

Decremps, F., Belliard, L., Perrin, B., and Gauthier, M.Sound Velocity and Absorption Measurements under High Pressure Using Picosecond Ultrasonics in a Diamond Anvil Cell: Application to the Stability Study of AlPdMn, (2008), Phys. Rev. Lett., 100:035502, 10.1103/PhysRevLett.100.035502.

Decremps, F., Gauthier, M., Ayrinhac, S., Bove, L., Belliard, L., Perrin, B., Morand, M., Marchand, G. L., Bergame, F., and Philippe, J.Picosecond acoustics method for measuring the thermodynamical properties of solids and liquids at high pressure and high temperature, (2015), Ultrasonics, 56:129 - 140, 
https://doi.org/10.1016/j.ultras.2014.04.011.

Dewaele, A., Belonoshko, A. B., Garbarino, G., Occelli, F., Bouvier, P., Hanfland, M., and Mezouar, M.High-pressure-high-temperature equation of state of $\mathrm{KCl}$ and KBr, (2012), Phys. Rev. B, 85:214105, 10.1103/PhysRevB.85.214105.

Dewaele, A., Loubeyre, P., Occelli, F., Mezouar, M., Dorogokupets, P. I., and Torrent, M.Quasihydrostatic Equation of State of Iron above 2 Mbar, (2006), Phys. Rev. Lett., 97:215504, 10.1103/PhysRevLett.97.215504.

Dorogokupets, P. I. and Oganov, A. R.Ruby, metals, and $\mathrm{MgO}$ as alternative pressure scales: A semiempirical description of shock-wave, ultrasonic, x-ray, and thermochemical data at high temperatures and pressures, (2007), Phys. Rev. B, 75:024115, 10.1103/PhysRevB.75.024115.

Dziewonski, A. M. and Anderson, D. L.Preliminary reference Earth model, (1981), Physics of the Earth and Planetary Interiors, 25(4):297 - 356, https://doi.org/10.1016/0031-9201(81)90046-7.

Fei, Y., Murphy, C., Shibazaki, Y., Shahar, A., and Huang, H.Thermal equation of state of hcp-iron: Constraint on the density deficit of Earth's solid inner core, (2016), Geophysical Research Letters, 43(13):6837-6843, 10.1002/2016GL069456.

Fischer, R. A., Campbell, A. J., Caracas, R., Reaman, D. M., Dera, P., and Prakapenka, V. B.Equation of state and phase diagram of Fe-16Si alloy as a candidate component of Earth's core, (2012), Earth and Planetary Science Letters, 357-358:268 - 276, https://doi.org/10.1016/j.epsl.2012.09.022.

Fischer, R. A., Campbell, A. J., Caracas, R., Reaman, D. M., Heinz, D. L., Dera, P., and Prakapenka, V. B.Equations of state in the Fe-FeSi system at high pressures and temperatures, (2014), Journal of Geophysical Research: Solid Earth, 119(4):2810-2827, 10.1002/2013JB010898.

Fischer, R. A., Campbell, A. J., Reaman, D. M., Miller, N. A., Heinz, D. L., Dera, P., and Prakapenka, V. B.Phase relations in the Fe-FeSi system at high pressures and temperatures, (2013), Earth and Planetary Science Letters, 373:5464, https://doi.org/10.1016/j.epsl.2013.04.035.

Fischer, R. A., Nakajima, Y., Campbell, A. J., Frost, D. J., Harries, D., Langenhorst, F., Miyajima, N., Pollok, K., and Rubie, D. C.High pressure metalsilicate partitioning of $\mathrm{Ni}, \mathrm{Co}, \mathrm{V}, \mathrm{Cr}, \mathrm{Si}$, and O, (2015), Geochimica et Cosmochimica Acta, 167:177 - 194, https://doi.org/10.1016/j.gca.2015.06.026.

Fitoussi, C., Bourdon, B., Kleine, T., Oberli, F., and Reynolds, B. C.Si isotope systematics of meteorites and terrestrial peridotites: implications for $\mathrm{Mg} / \mathrm{Si}$ fractionation in the solar nebula and for Si in the Earth's core, (2009), Earth and Planetary Science Letters, 287(1):77 - 85, https://doi.org/10.1016/ j.epsl.2009.07.038.

Hashin, Z. and Shtrikman, S.A variational approach to the theory of the elastic behaviour of polycrystals, (1962), Journal of the Mechanics and Physics of Solids, 10(4):343 - 352, https://doi.org/10.1016/0022-5096(62)90005-4.

Hirose, K., Morard, G., Sinmyo, R., Umemoto, K., Hernlund, J., Helffrich, G., and Labrosse, S.Crystallization of silicon dioxide and compositional evolution of the Earth's core, (2017), Nature, 543:99-.

Li, Y., Vočadlo, L., and Brodholt, J. P.The elastic properties of hcp-Fe alloys under the conditions of the Earth's inner core, (2018), Earth and Planetary Science Letters, 493:118 - 127, https://doi.org/10.1016/j.epsl.2018.04.013.

Lin, J.-F., Campbell, A. J., Heinz, D. L., and Shen, G.Static compression of ironsilicon alloys: Implications for silicon in the Earth's core, (2003a), Journal of Geophysical Research: Solid Earth, 108(B1):2045, 10.1029/2002JB001978.

Lin, J.-F., Struzhkin, V. V., Sturhahn, W., Huang, E., Zhao, J., Hu, M. Y., Alp, E. E., Mao, H.-k., Boctor, N., and Hemley, R. J.Sound velocities of iron-nickel and iron-silicon alloys at high pressures, (2003b), Geophysical Research Letters, 30(21):2112, 10.1029/2003GL018405. 
Litasov, K. D., Dorogokupets, P. I., Ohtani, E., Fei, Y., Shatskiy, A., Sharygin, I. S., Gavryushkin, P. N., Rashchenko, S. V., Seryotkin, Y. V., Higo, Y., Funakoshi, K., Chanyshev, A. D., and Lobanov, S. S.Thermal equation of state and thermodynamic properties of molybdenum at high pressures, (2013), Journal of Applied Physics, 113(9):093507, 10.1063/1.4794127.

Liu, J., Lin, J.-F., Alatas, A., Hu, M. Y., Zhao, J., and Dubrovinsky, L.Seismic parameters of hcp-Fe alloyed with $\mathrm{Ni}$ and $\mathrm{Si}$ in the Earth's inner core, (2016), Journal of Geophysical Research: Solid Earth, 121(2):610-623, 10.1002/2015JB012625.

Machová, A. and Kadečková, S.Elastic constants of iron-silicon alloy single crystals, (1977), Czechoslovak Journal of Physics B, 27(5):555-563, 10.1007/ BF01587133.

Mao, H. K., Xu, J., and Bell, P. M.Calibration of the ruby pressure gauge to 800 kbar under quasi-hydrostatic conditions, (1986), Journal of Geophysical Research: Solid Earth, 91(B5):4673-4676, 10.1029/JB091iB05p04673.

Mao, Z., Lin, J.-F., Liu, J., Alatas, A., Gao, L., Zhao, J., and Mao, H.-K.Sound velocities of Fe and Fe-Si alloy in the Earth's core, (2012), Proceedings of the National Academy of Sciences, 109(26):10239-10244, 10.1073/pnas.1207086109.

Martorell, B., Brodholt, J., G. Wood, I., and Vočadlo, L.The effect of nickel on the properties of iron at the conditions of Earth's inner core: Ab initio calculations of seismic wave velocities of Fe-Ni alloys, (2013), Earth and Planetary Science Letters, 365:143-151.

Martorell, B., Wood, I. G., Brodholt, J., and Vočadlo, L.The elastic properties of hcp-Fe1-xSix at Earth's inner-core conditions, (2016), Earth and Planetary Science Letters, 451:89 - 96, https://doi.org/10.1016/j.epsl.2016.07.018.

Masters, G. and Gubbins, D.On the resolution of density within the Earth, (2003), Physics of the Earth and Planetary Interiors, 140(1):159 - 167, https://doi.org/10.1016/j.pepi.2003.07.008. Geophysical and Geochemical Evolution of the Deep Earth.

McDonough, W. F. and Sun, S.-s.The composition of the Earth, (1995), Chemical Geology, 120(3):223 - 253, https://doi.org/10.1016/0009-2541(94)00140-4. Chemical Evolution of the Mantle.

Merkel, S., Hemley, R. J., and Mao, H.-k.Finite-element modeling of diamond deformation at multimegabar pressures, (1999), Applied Physics Letters, 74(5):656$658,10.1063 / 1.123031$.

Mezouar, M., Crichton, W. A., Bauchau, S., Thurel, F., Witsch, H., Torrecillas, F., Blattmann, G., Marion, P., Dabin, Y., Chavanne, J., Hignette, O., Morawe, C., and Borel, C.Development of a new state-of-the-art beamline optimized for monochromatic single-crystal and powder X-ray diffraction under extreme conditions at the ESRF, (2005), Journal of Synchrotron Radiation, 12(5):659-664, 10.1107/S0909049505023216.

Mezouar, M., Giampaoli, R., Garbarino, G., Kantor, I., Dewaele, A., Weck, G., Boccato, S., Svitlyk, V., Rosa, A. D., Torchio, R., Mathon, O., Hignette, O., and Bauchau, S.Methodology for in situ synchrotron X-ray studies in the laserheated diamond anvil cell, (2017), High Pressure Research, 37(2):170-180, 10.1080/08957959.2017.1306626.

Morard, G., Andrault, D., Antonangeli, D., Nakajima, Y., Auzende, A., Boulard, E., Cervera, S., Clark, A., Lord, O., Siebert, J., Svitlyk, V., Garbarino, G., and Mezouar, M.Fe-FeO and Fe-Fe3C melting relations at Earth's core-mantle boundary conditions: Implications for a volatile-rich or oxygen-rich core, (2017), Earth and Planetary Science Letters, 473:94 - 103, https://doi.org/ 10.1016/j.epsl.2017.05.024.

Morard, G., Andrault, D., Guignot, N., Siebert, J., Garbarino, G., and Antonangeli, D.Melting of $\mathrm{Fe}-\mathrm{Ni}-\mathrm{Si}$ and $\mathrm{Fe}-\mathrm{Ni}-\mathrm{S}$ alloys at megabar pressures: implications for the core-mantle boundary temperature, (2011), Physics and Chemistry of 
Minerals, 38(10):767-776, 10.1007/s00269-011-0449-9.

Morrison, R. A., Jackson, J. M., Sturhahn, W., Zhang, D., and Greenberg, E.Equations of state and anisotropy of Fe-Ni-Si alloys, (2018), Journal of Geophysical Research: Solid Earth, 123:4647 - 4675, https://doi.org/10.1029/ 2017JB015343.

Nguyen, J. H. and Holmes, N. C.Melting of iron at the physical conditions of the Earth's core, (2004), Nature, 427:339-.

Prescher, C. and Prakapenka, V. B.DIOPTAS: a program for reduction of twodimensional X-ray diffraction data and data exploration, (2015), High Pressure Research, 35(3):223-230, https://doi.org/10.1080/08957959.2015.1059835.

Ross, R. and Hume-Rothery, W.High temperature X-ray metallography: I. A new debye-scherrer camera for use at very high temperatures II. A new parafocusing camera III. Applications to the study of chromium, hafnium, molybdenum, rhodium, ruthenium and tungsten, (1963), Journal of the Less Common Metals, 5(3):258 - 270, https://doi.org/10.1016/0022-5088(63)90031-6.

Sakairi, T., Sakamaki, T., Ohtani, E., Fukui, H., Kamada, S., Tsutsui, S., Uchiyama, H., and Baron, A. Q.Sound velocity measurements of hcp Fe-Si alloy at high pressure and high temperature by inelastic X-ray scattering, (2018), American Mineralogist, 103(1):85, 10.2138/am-2018-6072.

Sakamaki, T., Ohtani, E., Fukui, H., Kamada, S., Takahashi, S., Sakairi, T., Takahata, A., Sakai, T., Tsutsui, S., Ishikawa, D., Shiraishi, R., Seto, Y., Tsuchiya, T., and Baron, A. Q. R.Constraints on Earth's inner core composition inferred from measurements of the sound velocity of hcp-iron in extreme conditions, (2016), Science Advances, 2(2):e1500802, 10.1126/sciadv.1500802.

Siebert, J., Badro, J., Antonangeli, D., and Ryerson, F. J.Terrestrial Accretion Under Oxidizing Conditions, (2013), Science, 339(6124):1194-1197, 10.1126/science.1227923.

Sokolova, T., Dorogokupets, P., and Litasov, K.Self-consistent pressure scales based on the equations of state for ruby, diamond, $\mathrm{MgO}, \mathrm{B} 2-\mathrm{NaCl}$, as well as $\mathrm{Au}$, $\mathrm{Pt}$, and other metals to $4 \mathrm{Mbar}$ and $3000 \mathrm{~K}$, (2013), Russian Geology and Geophysics, 54(2):181 - 199, https://doi.org/10.1016/j.rgg.2013.01.005.

Tateno, S., Kuwayama, Y., Hirose, K., and Ohishi, Y.The structure of FeSi alloy in Earth's inner core, (2015), Earth and Planetary Science Letters, 418:11 - 19, https://doi.org/10.1016/j.epsl.2015.02.008.

Tsuchiya, T. and Fujibuchi, M.Effects of Si on the elastic property of Fe at Earth's inner core pressures: First principles study, (2009), Physics of the Earth and Planetary Interiors, 174:212-219, 10.1016/j.pepi.2009.01.007.

Václav, P., Michal, D., and Lukáš, P. Crystallographic Computing System JANA2006: General features.

Vinet, P., Rose, J. H., Ferrante, J., and Smith, J. R.Universal features of the equation of state of solids, (1989), Journal of Physics: Condensed Matter, 1(11):1941.

Vočadlo, L.Ab initio calculations of the elasticity of iron and iron alloys at inner core conditions: Evidence for a partially molten inner core?, (2007), Earth and Planetary Science Letters, 254(1):227 - 232, https://doi.org/10.1016/ j.epsl.2006.09.046.

Vočadlo, L., Alfè, D., Gillan, M., and Price, G.The properties of iron under core conditions from first principles calculations, (2003), Physics of the Earth and Planetary Interiors, 140(1):101 - 125, https://doi.org/10.1016/ j.pepi.2003.08.001. Geophysical and Geochemical Evolution of the Deep Earth.

Wade, J. and Wood, B.Core formation and the oxidation state of the Earth, (2005), Earth and Planetary Science Letters, 236(1):78 - 95, https://doi.org/10.1016/ j.epsl.2005.05.017. 
Wakamatsu, T., Ohta, K., Yagi, T., Hirose, K., and Ohishi, Y.Measurements of sound velocity in iron-nickel alloys by femtosecond laser pulses in a diamond anvil cell, (2018), Physics and Chemistry of Minerals, -:1-7, 10.1007/s00269-018-0944-3.

Wood, B. J., Bryndzia, L. T., and Johnson, K. E.Mantle Oxidation State and Its Relationship to Tectonic Environment and Fluid Speciation, (1990), Science, 248(4953):337-345, 10.1126/science.248.4953.337.

Yamazaki, D., Ito, E., Yoshino, T., Yoneda, A., Guo, X., Zhang, B., Sun, W., Shimojuku, A., Tsujino, N., Kunimoto, T., Higo, Y., and Funakoshi, K.-i.P-V-T equation of state for epsilon-iron up to $80 \mathrm{GPa}$ and $1900 \mathrm{~K}$ using the Kawaitype high pressure apparatus equipped with sintered diamond anvils, (2012), Geophysical Research Letters, 39(20):L20308, 10.1029/2012GL053540. 